\title{
Transforming Acidic Coiled-coil Protein-3: A Novel Marker for Differential Diagnosis and Prognosis Prediction in Endocervical Adenocarcinoma
}

\section{YanLin Wen}

Sun Yat-sen University Cancer Center https://orcid.org/0000-0003-0109-2915

\section{Shu-Mei Yan}

Sun Yat-sen University Cancer Center

\section{Wei Wei}

Sun Yat-sen University Cancer Center

\section{Xia Yang}

Sun Yat-sen University Cancer Center

\section{Shi-Wen Zhang}

Sun Yat-sen University Cancer Center

Jing-Ping Yun

Sun Yat-sen University Cancer Center

\section{Li-Li Liu}

Sun Yat-sen University Cancer Center

Rong-Zhen Luo ( $\sim$ luorzh@sysucc.org.cn )

Sun Yat-sen University Cancer Center https://orcid.org/0000-0002-6599-0370

\section{Research article}

Keywords: TACC3, ECA, HPVA, NHPVA, prognosis

Posted Date: September 18th, 2020

DOI: https://doi.org/10.21203/rs.3.rs-77511/v1

License: (a) This work is licensed under a Creative Commons Attribution 4.0 International License. Read Full License

Version of Record: A version of this preprint was published at Molecular Medicine on June 10th, 2021. See the published version at https://doi.org/10.1186/s10020-021-00298-z. 


\section{Abstract}

\section{Background}

Endocervical adenocarcinoma (ECA) is classified as human papilloma virus (HPV)-associated (HPVA) or non-HPVA (NHPVA) type in the International Endocervical Adenocarcinoma Criteria and Classification (IECC). Of them, HPVA is recognized as the representative ECA subtype, which is related to typical molecular characteristics. Transforming acidic coiled-coil protein-3 (TACC3) is an oncogene with frequent abnormal expression, and it also represents a vital biomarker for various human malignancies. This work aimed to examine the role of TACC3 in the diagnosis and prognosis of ECA.

Methods

We analyzed 264 ECA patients who underwent surgical resection, and tumors were classified into HPVA and NHPVA subtypes. The expression levels of TACC3, P16, MLH1, PMS2, MSH2, MSH6, Ki-67 in tumor were evaluated on tissue microarray by immunohistochemistry (IHC). HPV subtypes were detected on formalin-fixed paraffin-embedded (FFPE) ECA tissues by polymerase chain reaction (PCR).

Results

As suggested by IHC, ECA samples showed increased TACC3 level relative to related non-carcinoma samples. TACC3 expression was also elevated in HPVA compared with NHPA. In HPVA subtype, high TACC3 expression was significantly correlated with P16-positive, Ki-67-high expression. Furthermore, TACC3 level was remarkably related to the histological type $(P=0.006)$, nerve invasion $(P=0.003)$, differentiation ( $P=0.004)$, surgical margin $(P=0.012)$, parametrium invasion $(P=0.040)$, Ki-67 $(P=0.004)$ and P16 $(P=0.000)$. Besides, upon Kaplan-Meier analysis, the up-regulated TACC3 was tightly related to the poor overall survival (OS, $P=0.001)$, disease-free survival (DFS, $P<0.001)$ as well as recurrence survival $(P<0.001)$. Moreover, upon multivariate analysis, the elevated TACC3 level served as the marker to independently predict ECA prognosis. ROC curve analyses indicate that TACC3, P16 and HPV subtype showed similar results for distinguishing HPVA from NHPVA, with an area under the ROC curve of 0.640 , 0.649 and 0.675 , respectively. The combination of TACC3 and HPV subtypes improved diagnostic performance for ECA compared with TACC3, P16 and HPV subtype alone.

\section{Conclusions}

Taken together, findings in this work reveal that TACC3 may be used as the new biomarker for the diagnosis and prognosis of ECA.

\section{Introduction}

The International Endocervical Adenocarcinoma Criteria and Classification (IECC) represents the updated endocervical adenocarcinoma (ECA) classification approach, which is suggested to replace the classification method of WHO(Ward et al.,2012, Stolnicu et al.,2018, Stolnicu et al.,2019b). According to 
IECC, ECA can be classified based on the morphological characteristics associated with the infection of human papilloma virus (HPV) into HPV-associated adenocarcinoma (HPVA) or non-HPV-associated adenocarcinoma (NHPVA). Of them, the apical apoptosome and mitotic bodies can be easily recognized form the HPVA subtype(Hodgson et al.,2019b). Recent studies have shown that clinical and demographic parameters differ between HPVA and NHPVA. NHPVA has an aggressive phenotype and distinct molecular features (Stolnicu et al.,2019a, Karamurzin et al.,2015). It is necessary to find the more creditable biomarkers to predict the diagnosis and prognosis of ECA, so as to provide superior therapeutic strategies for ECA cases.

Transforming acidic coiled-coil protein 3 (TACC3), one of the TACC family members, can be encoded by TACC3 gene in 4P16.3(He et al.,2016). As one of the spindle regulatory proteins, TACC3 has a conserved TACC domain at the C-terminal, which plays an important role in its alignment with tubulins and in promoting effective elongation of tubules during mitosis(Gergely et al.,2000, Ha, Kim and Breuer,2013b, Sun et al.,2017). As one of the spindle regulatory proteins, TACC3 has a conserved TACC domain at the Cterminal, which plays an important role in its alignment with tubulins and in promoting effective elongation of tubules during mitosis(Piekorz et al.,2002, Mahdipour et al.,2015). More and more studies indicate that, the abnormal expression of TACC3 possibly plays a role of the oncogene, leading to multipole spindle formation, cell cycle arrest, cell death, and epithelial-mesenchymal transformation (EMT)(Ha, Park and Breuer,2013c, Huang et al.,2015, Peters et al.,2005, Song et al.,2018, Yun et al.,2015). Amounting researches have indicated that high TACC3 is overexpressed in various solid tumors (Bhosale et al.,2016), such as ovarian cancer(Lauffart et al.,2005), glioblastoma(Duncan et al.,2010), esophageal squamous cell carcinoma(Huang et al.,2015), hepatocellular carcinoma(Nahm et al.,2016, Zhou et al.,2015), gastric carcinoma(Yun et al.,2015) and non-small cell lung cancer(Jung et al.,2006). FGFR3TAAC3, the TACC3 fusion gene, promotes cancer cell development in some cancer types by promoting cell proliferation(Parker et al.,2013, Capelletti et al.,2014, Yuan et al.,2014, Du et al.,2016). However, the clinical significance of TACC3 in different histologic types of ECA has not yet been reported.

However, the clinical significance of TACC3 in different histologic types of ECA has not yet been reported.

\section{Materials And Methods}

\section{Patients and samples}

The Institutional Ethical Boards of Sun Yat-sen University Cancer Center approved our study protocol. The documented pathological specimens embedded in paraffin (obtained from January 2010 and December 2014) were acquired from 264 cases with primary ECA in both histological subtypes for analysis, including 239 HPVA and 25 HPVA cases. At the same time, the pathological and clinical data from these patients were collected from the patient records. For our enrolled patients, their age was 19-76 (average, 65.4) months, with the follow-up period of 65.4 months.

Tissue microarray (TMA) construction and immunohistochemistry (IHC) 
Tumorous tissue and adjacent nontumorous ECA tissues were constructed for TMA. TMA block was sliced to the 4- $\mu \mathrm{m}$ sections, followed by IHC staining. Afterwards, each slide was deparaffinized with xylene and ethanol, followed by $3 \%$ hydrogen peroxide treatment within methanol. Then, the slides were blocked with avidin-biotin under $4^{\circ} \mathrm{C}$ overnight, followed by incubation with antibodies for TACC3 (ab134154, Abcam), P16 (Roche, Germany), MLH1 (Roche (M1), Germany), PMS2 (Dako (EP51), Germany), MSH2 (ZA0622, Zhongshan, China), MSH6 (Roche (SP93), Germany) and Ki-67 (ZA0502, Zhongshan, China). Later, slides were washed by PBS thrice, and further incubated using the biotinylated goat anti-mouse antibodies, followed by DAKO liquid 3,3'-diaminobenzidine tetrahydro-chloride (DAB) staining as well as Mayer's hematoxylin counterstaining. Two experienced pathologists evaluated the staining. In the presence of block-like, diffuse staining in each core positive P16 was defined, while patchy or no staining indicated negative staining. MSH2/MSH6/PMS2/MLH1 was interpreted as positive if $\geq 1 \%$ of tumor cell nuclei were positive. For samples with positive TACC3 staining, the scores were rated as follows, $0,<5 \%$ cells with positive staining; $1,5 \%-24 \%$ cells with positive staining; $2,25 \%-49 \%$ cells with positive staining; $3,50 \%-74 \%$ cells with positive staining; $4,75 \%-100 \%$ cells with positive staining. In addition, the intensity of positive staining was rated below: 0 , negative; 1 , weak; 2 , moderate; and 3 , strong staining. As for the final score, it was determined through percentage score multiplied by intensity score. The best cut-off values for all variables were determined by X-tile (Camp RL, Clin Cancer Res, 2004) and were as follows: age (37 years), tumor size $(4.5 \mathrm{~cm})$, TACC3 (3.7), Ki-67 (12.5\%).

\section{HPV subtypes}

Tumor tissue for PCR testing was attempted in tumors not represented in tissue microarrays, as described in the previous study (Hodgson et al.,2019a). The Roche Cobas 4800 system (Pleasanton, CA) was utilized for HPV detection, which evaluates for the presence of 14 types of the following HPV DNA: 16, 18, $31,33,35,39,45,51,52,56,58,59,66$, and 68 .

\section{Statistical analysis}

SPSS 25.0 (SPSS, Chicago, IL, USA) was employed for statistical analysis. Significant differences in the expression of TACC3 were determined by student's t-test. Data displayed within the bar and column charts were expressed in the manner of mean \pm SEM. Associations of TACC3 expression with clinicopathological characteristics were analyzed through Fisher's exact test or Pearson's chi-square test. The scatter plot shows the correlation between TACC3 and P16 and Ki-67. In addition, the receiver operating characteristics (ROC) curves were plotted and the areas under curve (AUC) values together with the corresponding $95 \%$ confident interval $(95 \% \mathrm{Cl})$ were obtained to evaluate the sensitivity and specificity. The Kaplan-Meier analysis was adopted to analyze survival, and survival-related factors were discovered upon the Cox proportional hazard regression model. A difference of $\mathrm{P}<0.05$ indicated statistical significance.

\section{Results}


Altogether 264 ECA cases undergoing resection for primary tumor were enrolled into the present work. Based on IHC analysis, TACC3 positive staining rates within tumor and non-carcinoma samples were $61.4 \%$ and $13.1 \%$, separately. Besides, the over-expression of TACC3 is found to facilitate tumor genesis and development. Weak, moderate and strong staining was seen in typical IHC images regarding TACC3 levels within tumor samples (Figure 1A). TACC3 was mainly distributed within cytoplasm, and its level in ECA significantly increased relative to non-carcinoma samples (Figure 1B and C), in agreement with The Cancer Genome Atlas (TCGA) data (Supplementary Figure 1A). As found in the other TMA cohort enrolling 30 ECA patients who developed lymph nodes metastasis (LNM), no increased TACC3 immunoreactivity was measured within LNM lesions relative to related primary counterparts, consistent with TCGA data (Supplementary Figure 1B). As shown by the IHC data, there was no significantly different between the lymph nodes metastases and the primary lesions (Figure 1D and E). Collectively, findings in this study suggested the over-expression of TACC3 in ECA relative to the non-carcinoma samples.

\section{TACC3, P16 and Ki-67 expression in HPVA and NHPVA}

Furthermore, we confirm the expression profile of TACC3 in different histologic subtypes of ECA. Supplementary Table 1 presents the clinicopathological data for cases of 2 ECA histological subtypes. According to the IHC data, the positive staining of TACC3 in HPVA and NHPVA were $64.0 \%, 36.0 \%$, respectively. The data suggest TACC3 overexpression may perform its diverse biological functions in different histological subtypes. Representative IHC images for TACC3, P16 and Ki-67 in HPVA and NHPVA subtypes are shown in Figure 2A. TACC3 expression in HPVA was remarkably higher than that in NHPVA (Figure 2B). Positive associations were found between high TACC3 expression and P16 positive expression, high TACC3 expression and high $\mathrm{Ki}-67$ expression in HPVA (R2=0.036, $\mathrm{P}=0.003 ; \mathrm{R} 2=0.140$, $P<0.001$, respectively), but no significantly associations were found in NHPA (Figure $2 C$ and $D$ ). The representative IHC images of TACC3 expression in good-, moderate- and low-differentiation tumor (Figure 2E). TACC3 expression in low-differentiation tumor was remarkably higher than that in gooddifferentiation (Figure 2F), in agreement with TCGA data (Supplementary Figure 1C).

\section{Impact of TACC3 on the overall survival (OS) and disease-free survival (DFS) of ECA patients}

For better determining the clinical implication of TACC3 for ECA, the associations of TACC3 level with clinicopathological factors for ECA cases were examined. All ECA patients were classified as 2 groups based on IHC staining score threshold of 3.7 for TACC3, including high or low TACC3 level group (Supplementary Figure2A). High TACC3 level was detected in $61.4 \%(162 / 264)$ patients. Upon statistical analysis, the increased TACC3 level was related to differentiation $(P=0.004)$, histological type $(P=0.006)$, parametrium invasion $(P=0.040)$, nerve invasion $(P=0.003)$, surgical margin $(P=0.012)$, Ki-67 $(P=0.004)$ and P16 ( $P=0.000)$ (Table 1). Similarly, TCGA data showed that TACC3 expression was associated with proliferation related parameters, such as E2F targets, G2M checkpoint, G2 pathway and proliferationassociated biomarker (Supplementary Figure 1D-F and Supplementary Figure 2B). Afterwards, the value of TACC3 in predicting the prognosis for ECA was analyzed. As suggested by Kaplan-Meier results, cases 
having high TACC3 level were associated with reduced OS $(P=0.001)$, as well as OS at $1(P=0.021), 3$ $(P=0.011)$ and 5 years $(P=0.011)$ relative to those with low TACC3 expression. Besides, the high TACC3 level was related to dismal DFS $(P<0.001)$ as well as the recurrence tendency $(P<0.001)$ of ECA cases (Figure 3). Furthermore, the stratified survival analysis verified that TACC3 showed prognostic significance. It was also shown that, the TACC3 level was related to various OS-related pathological factors (Figure 4). As a result, the present work indicated that, TACC3 might predict ECA prognosis.

Cox regression analysis showed that TACC3 expression was identified as a prognostic factor, along with age, FIGO stage, tumor size, histologic type, stromal invasion, nerves invasion, LVI, LNM, parametrium invasion, surgical margin and P16 expression (Table 2). The multivariate survival analysis suggested that TACC3 as an independent factor for worse OS (HR=2.280, 95\%Cl: 1.087-4.783, $\mathrm{P}=0.029)$ and poor DFS $(\mathrm{HR}=2.265,95 \% \mathrm{Cl}: 1.232-4.166, \mathrm{P}=0.009)$ (Table 2 and 3$)$. On the whole, this study suggested the potential of TACC3 as the biomarker to independently predict ECA prognosis.

\section{Diagnostic performance of TACC3 in ECA compared to other detection methods.}

TACC3 and P16 protein detected by IHC, HPV subtypes detected by PCR were used as detection methods in our study to distinguish HPVA from NHPVA. The positive rates of TACC3, P16 and HPV subtypes were $61.4 \%, 90.9 \%$ and $59.7 \%$, respectively (Figure $5 A$ ). ROC curve analyses suggest that TACC3, P16 and HPV subtypes showed similar results for distinguishing HPVA from NHPA (AUC $=0.640$, sensitivity $=64.0 \%$, specificity $=64.0 \%$; AUC $=0.649$, sensitivity $=93.7 \%$, specificity $=36.0 \%$; $A U C=0.675$, sensitivity $=63.0 \%$, specificity $=72.0 \%$, respectively) (Supplementary Table 2 ). ROC curve analyses showed that combination of TACC3 and HPV subtypes improved diagnostic performance for ECA compared with TACC3, P16 and HPV subtypes alone (Figure 5B and Supplementary Table 2). The correlation of TACC3, P16 and HPV subtypes was also studied (Figure 5C and D). In our study, we find $60 \%(157 / 263)$ of ECA are associated with HPV, particularly with HPV strains 16 and 18. In different HPV subtypes, the positivity of P16 for the diagnosis of ECA was much higher compared to TACC3 (Figure $5 \mathrm{C}$ ). Our results also show that the positivity of TACC3 in all cases and HPVA showing p16 protein detected by IHC (All case, 65.8\%; HPVA, $67.4 \%$ ), similar with the positivity of HPV subtypes (All case, 63.3\%; HPVA, 65.6\%). The positivity of TACC3 and HPV subtypes was much higher in P16-positive group compared to that in P16-negative group (Figure 5D). Therefore, our data indicated TACC3 may be as a diagnostic marker for ECA patients.

\section{Discussion}

Currently, different from cervical squamous cell carcinoma (CSCC), ECA is relatively rare, and it is not usually related to HPV infection(RJ et al.,2014, Park et al.,2011). According to the IECC, ECAs can be classified as HPVA or NHPVA subtype based on only the HE slides. As for these two major IECC subtypes, they are different in terms of demographic and clinical features. For improving ECA prognostic outcomes and better stratifying HPVA and NHPVA, it is necessary to reveal the possible molecular mechanism, so as to discover the more creditable biomarkers for disease diagnosis and prognosis. ECA is the malignancy with rapid growth, so this study aimed to examine the effect of spindle regulatory proteins on 
the development of ECA. According to our results, TACC3, one of the spindle regulatory proteins, showed over-expression in ECA, which was tightly related to the poor OS and DFS. Moreover, multivariate analysis revealed that TACC3 might serve as the biomarker to independently predict prognosis. Additionally, TACC3 was also used to be the biomarker to diagnose ECA. As a result, the present work first unveiled the clinical implication TACC3 for ECA.

The abnormal TACC3 expression is detected in human malignancies, and its role has been extensively investigated(Wang et al.,2017). TACC3 is over-expressed in human malignancies and demonstrates the oncogenic property(Wang et al.,2017). In addition, TACC3 over-expression is identified to be related to dismal prognostic outcome(Song et al.,2018, Li et al.,2017, Nahm et al.,2016). TACC3 is previousy suggested to exert a vital part in the EGF-mediated EMT, which represents a promising therapeutic to treat cervical carcinoma and is involved in the EGF/EGFR receptor signal transduction pathway(Ha, Kim and Breuer,2013a). Nonetheless, its expression pattern and clinical value for cancer remain largely unknown. In the present work, the TACC3 level was up-regulated in 264 ECA cases. ECA cases showing up-regulated TACC3 expression were associated with the shortened OS and DFS relative to those with down-regulated expression. The above results indicate that TACC3 possibly served as the new biomarker to predict the prognosis for HCC cases. In addition, TACC3 protein level was up-regulated in moderately- to poorly differentiated samples relative to the well-differentiated samples. As a result, it is necessary to examine the role of TACC3 in the differentiation of ECA cells. A number of articles have attempted to examine the role of TACC3 in tumor development, yet the underlying molecular mechanisms regarding tumor development are still unknown. As discovered by Hyoung et al., TACC3 activated the PI3/AKT and ERK signal transduction pathways to accelerate the epithelial-mesenchymal transition (EMT) process(Ha et al.,2013c). Besides, it is recently suggested that, the TACC3 level decreases by HDACls, and the specific TACC3 knockdown inhibits the proliferation and clone forming capacity of CCA cells(He et al.,2016). TACC3 also facilitates chemosensitivity of the breast cancer cells (Schneider et al.,2008, Schmidt et al.,2010, Yim et al.,2009). TACC3 knockdown improves tumor cell sensitivity to chemotherapeutics through the effective regulation of premature senescence(Yim et al.,2009). For enhancing the efficacy for treating ECA, more studies should be done to develop the systemic therapeutic strategies. Hopefully, targeted therapies associated with TACC 3 can be realized in the future.

Paclitaxel is suggested in prior research to have antitumor effect on TACC3 effective within the cervical cancer cells with positive HPV-18 infection(Yim et al.,2009). The TACC3 protein levels were up-regulated in HPVA tissues relative to NHPVA ones. As a result, it is important to examine the role of TACC 3 in the development of HPVA. IHC against P16 protein, DNA PCR, and DNA- or RNA-targeting in situ hybridization are the most commonly used HPV tests. In our study, we used PCR and IHC against P16 protein to assess HPV status. PCR, the gold-standard test for assessing the HPV status, detects HPV within a malignancy and determines the related genotype(Cheung et al.,2019). Besides, P16 immunostaining represents the indirect, cost-effective marker for viral infection, which has been extensively utilized in practical applications. It shows the highest sensitivity but low specificity, because the over-expression of P16 is detected in any situation with up-regulated cell cycle (inflammation or other viral infections)(Nuovo et al.,1999). In the ROC curve, P16 immunostaining had the best diagnostic effect, while TACC3 IHC and 
HPV Subtype had similar diagnostic effect. However, TACC3 IHC and HPV subtype could be combined to improve the diagnostic effectiveness. When both P16 IHC and HPV Subtype were negative, TACC3 might be positively expressed. In this case, the missed diagnosis of endocervical adenocarcinoma could be avoided to a certain extent, especially in the case of biopsy. Therefore, TACC3 is expected to be a new immunohistochemical marker for detection of ECA.

To sum up, findings in this work demonstrate the up-regulation of TACC3 within the clinical ECA samples. The down-regulated TACC3 expression is found to predict poor OS. All in all, this work identifies that TACC3 may be used to be the potential biomarker to diagnose and predict ECA prognosis.

\section{Declarations}

Financial support: The National Natural Science Foundation of China (No. 81872012, 81872266). The National Natural Science Foundation of Guangdong province (No.2018B030311005) and Science and Technology Program of Guangzhou (No.201707020038). The authenticity of this article has been validated by uploading the key raw data onto the Research Data Deposit public platform (www.researchdata.org.cn) with the approval RDD number RDDB2020000652.

Contributors Conception and design: Rong-Zhen Luo. Performing experiments: Shu-Mei Yan and Xia Yang. Drafting of the article: Li-Li Liu and Yan-Lin Wen. Acquisition and interpretation of data, review, editing and approval of the manuscript: all authors.

\section{Disclosure of conflict of interest}

None.

\section{References}

Bhosale P, Ma J, lyer R, et al.Feasibility of a reduced field-of-view diffusion-weighted ( $r F O V$ ) sequence in assessment of myometrial invasion in patients with clinical FIGO stage I endometrial cancer. J Magn Reson Imaging.2016; 43(2):316-24.

Capelletti M, Dodge M E, Ercan D, et al.Identification of recurrent FGFR3-TACC3 fusion oncogenes from lung adenocarcinoma. Clin Cancer Res.2014; 20(24):6551-8.

Cheung T H, Yim S F, Yu M Y, et al.Liquid biopsy of HPV DNA in cervical cancer. J Clin Virol.2019; 114:3236.

Du Y, Liu L, Wang C, et al.TACC3 promotes colorectal cancer tumourigenesis and correlates with poor prognosis. Oncotarget.2016; 7(27):41885-41897.

Duncan C G, Killela P J, Payne C A, et al.Integrated genomic analyses identify ERRFI1 and TACC3 as glioblastoma-targeted genes. Oncotarget.2010; 1(4):265-77. 
Gergely F, Karlsson C, Still I, et al.The TACC domain identifies a family of centrosomal proteins that can interact with microtubules. Proc Natl Acad Sci U S A.2000; 97(26):14352-7.

Ha G H, Kim J L \& Breuer E K.TACC3 is essential for EGF-mediated EMT in cervical cancer. PLoS One.2013a; 8(8):e70353.

--.Transforming acidic coiled-coil proteins (TACCs) in human cancer. Cancer Lett.2013b; 336(1):24-33.

Ha G H, Park J S \& Breuer E K.TACC3 promotes epithelial-mesenchymal transition (EMT) through the activation of PI3K/Akt and ERK signaling pathways. Cancer Lett.2013c; 332(1):63-73.

He J C, Yao W, Wang J M, et al.TACC3 overexpression in cholangiocarcinoma correlates with poor prognosis and is a potential anti-cancer molecular drug target for HDAC inhibitors. Oncotarget.2016; 7(46):75441-75456.

Hodgson A, Howitt B E, Park K J, et al.Genomic Characterization of HPV-related and Gastric-type Endocervical Adenocarcinoma: Correlation With Subtype and Clinical Behavior. Int J Gynecol Pathol.2019a.

Hodgson A, Park K J, Djordjevic B, et al.International Endocervical Adenocarcinoma Criteria and Classification: Validation and Interobserver Reproducibility. Am J Surg Pathol.2019b; 43(1):75-83.

Huang Z L, Lin Z R, Xiao Y R, et al.High expression of TACC3 in esophageal squamous cell carcinoma correlates with poor prognosis. Oncotarget.2015; 6(9):6850-61.

Jung $\mathrm{C} \mathrm{K}$, Jung $\mathrm{J} \mathrm{H}$, Park $\mathrm{G}$ S, et al.Expression of transforming acidic coiled-coil containing protein 3 is a novel independent prognostic marker in non-small cell lung cancer. Pathol Int.2006; 56(9):503-9.

Karamurzin Y S, Kiyokawa T, Parkash V, et al.Gastric-type Endocervical Adenocarcinoma: An Aggressive Tumor With Unusual Metastatic Patterns and Poor Prognosis. Am J Surg Pathol.2015; 39(11):1449-57.

Lauffart B, Vaughan M M, Eddy R, et al.Aberrations of TACC1 and TACC3 are associated with ovarian cancer. BMC Womens Health.2005; 5:8.

Li Q, Ye L, Guo W, et al.Overexpression of TACC3 is correlated with tumor aggressiveness and poor prognosis in prostate cancer. Biochem Biophys Res Commun.2017; 486(4):872-878.

Mahdipour M, Leitoguinho A R, Zacarias Silva R A, et al.TACC3 Is Important for Correct Progression of Meiosis in Bovine Oocytes. PLoS One.2015; 10(7):e0132591.

Nahm J H, Kim H, Lee H, et al.Transforming acidic coiled-coil-containing protein 3 (TACC3) overexpression in hepatocellular carcinomas is associated with "stemness" and epithelial-mesenchymal transition-related marker expression and a poor prognosis. Tumour Biol.2016; 37(1):393-403. 
Nuovo G J, Plaia T W, Belinsky S A, et al.In situ detection of the hypermethylation-induced inactivation of the p16 gene as an early event in oncogenesis. Proc Natl Acad Sci U S A.1999; 96(22):12754-9.

Park K J, Kiyokawa T, Soslow R A, et al.Unusual endocervical adenocarcinomas: an immunohistochemical analysis with molecular detection of human papillomavirus. Am J Surg Pathol.2011; 35(5):633-46.

Parker B C, Annala M J, Cogdell D E, et al.The tumorigenic FGFR3-TACC3 gene fusion escapes miR-99a regulation in glioblastoma. J Clin Invest.2013; 123(2):855-65.

Peters D G, Kudla D M, Deloia J A, et al.Comparative gene expression analysis of ovarian carcinoma and normal ovarian epithelium by serial analysis of gene expression. Cancer Epidemiol Biomarkers Prev.2005; 14(7):1717-23.

Piekorz R P, Hoffmeyer A, Duntsch C D, et al.The centrosomal protein TACC3 is essential for hematopoietic stem cell function and genetically interfaces with p53-regulated apoptosis. EMBO J.2002; 21(4):653-64.

RJ K, ML C, CS H, et al.WHO Classification of Tumors of Female Reproductive Organs, 4th ed. Lyon: IARC, WHO Press.2014.

Schmidt S, Schneider L, Essmann F, et al.The centrosomal protein TACC3 controls paclitaxel sensitivity by modulating a premature senescence program. Oncogene.2010; 29(46):6184-92.

Schneider L, Essmann F, Kletke A, et al.TACC3 depletion sensitizes to paclitaxel-induced cell death and overrides p21WAF-mediated cell cycle arrest. Oncogene.2008; 27(1):116-25.

Song H, Liu C, Shen N, et al.Overexpression of TACC3 in Breast Cancer Associates With Poor Prognosis. Appl Immunohistochem Mol Morphol.2018; 26(2):113-119.

Stolnicu S, Barsan I, Hoang L, et al.Stromal invasion pattern identifies patients at lowest risk of lymph node metastasis in HPV-associated endocervical adenocarcinomas, but is irrelevant in adenocarcinomas unassociated with HPV. Gynecol Oncol.2018; 150(1):56-60.

Stolnicu S, Hoang L, Chiu D, et al.Clinical Outcomes of HPV-associated and Unassociated Endocervical Adenocarcinomas Categorized by the International Endocervical Adenocarcinoma Criteria and Classification (IECC). Am J Surg Pathol.2019a; 43(4):466-474.

Stolnicu S, Hoang L, Hanko-Bauer O, et al.Cervical adenosquamous carcinoma: detailed analysis of morphology, immunohistochemical profile, and clinical outcomes in 59 cases. Mod Pathol.2019b; 32(2):269-279.

Sun Y, Tian Y, Wang G Z, et al.Overexpression of Transforming Acidic Coiled CoilContaining Protein 3 Reflects Malignant Characteristics and Poor Prognosis of Glioma. Int J Mol Sci.2017; 18(3). 
Wang J, Du S, Fan W, et al.TACC3 as an independent prognostic marker for solid tumors: a systematic review and meta-analysis. Oncotarget.2017; 8(43):75516-75527.

Ward K K, Shah N R, Saenz C C, et al.Changing demographics of cervical cancer in the United States (1973-2008). Gynecol Oncol.2012; 126(3):330-3.

Yim E K, Tong S Y, Ho E M, et al.Anticancer effects on TACC3 by treatment of paclitaxel in HPV-18 positive cervical carcinoma cells. Oncol Rep.2009; 21(2):549-57.

Yuan L, Liu Z H, Lin Z R, et al.Recurrent FGFR3-TACC3 fusion gene in nasopharyngeal carcinoma. Cancer Biol Ther.2014; 15(12):1613-21.

Yun M, Rong J, Lin Z R, et al.High expression of transforming acidic coiled coil-containing protein 3 strongly correlates with aggressive characteristics and poor prognosis of gastric cancer. Oncol Rep.2015; 34(3):1397-405.

Zhou D S, Wang H B, Zhou Z G, et al.TACC3 promotes stemness and is a potential therapeutic target in hepatocellular carcinoma. Oncotarget.2015; 6(27):24163-77.

\section{Tables}

Table 1. Correlation of clinicopathological parameters and TACC3 expression $(n=264)$. 


\begin{tabular}{|c|c|c|c|c|}
\hline \multirow[t]{2}{*}{ Variable } & \multicolumn{4}{|c|}{ TACC3 expression } \\
\hline & All cases & Low & High & $P$ value $^{\mathrm{a}}$ \\
\hline Age (years) & & & & 0.114 \\
\hline$<37$ & 31 & $16(51.6 \%)$ & $15(48.4 \%)$ & \\
\hline$\geq 37$ & 233 & $86(36.9 \%)$ & $147(63.1 \%)$ & \\
\hline Figo stage & & & & 0.085 \\
\hline I & 186 & $66(35.5 \%)$ & $120(64.5 \%)$ & \\
\hline$\|$ & 67 & $30(44.8 \%)$ & $37(55.2 \%)$ & \\
\hline III & 8 & $3(37.5 \%)$ & $5(62.5 \%)$ & \\
\hline IV & 3 & $3(100.0 \%)$ & $0(0.0 \%)$ & \\
\hline Tumor size(cm) & & & & 0.414 \\
\hline$<4.5$ & 221 & $83(37.6 \%)$ & $138(62.4 \%)$ & \\
\hline$\geq 4.5$ & 43 & $19(44.2 \%)$ & $24(55.8 \%)$ & \\
\hline Histological type & & & & 0.006 \\
\hline HPVA & 239 & $86(36.0 \%)$ & $153(64.0 \%)$ & \\
\hline NHPVA & 25 & $16(64.0 \%)$ & $9(36.0 \%)$ & \\
\hline Differentiation & & & & 0.004 \\
\hline Good & 11 & $8(72.7 \%)$ & $3(27.3 \%)$ & \\
\hline Moderate & 140 & $61(43.6 \%)$ & $79(56.4 \%)$ & \\
\hline Low & 113 & $33(29.2 \%)$ & $80(70.8 \%)$ & \\
\hline Stromal invasion & & & & 0.120 \\
\hline$<1 / 3$ & 67 & $27(40.3 \%)$ & $40(59.7 \%)$ & \\
\hline $1 / 3-2 / 3$ & 81 & $24(29.6 \%)$ & $57(70.4 \%)$ & \\
\hline$\geq 2 / 3$ & 116 & $50(43.1 \%)$ & $66(56.9 \%)$ & \\
\hline Nerve invasion & & & & 0.003 \\
\hline Negative & 238 & $85(35.7 \%)$ & $153(64.3 \%)$ & \\
\hline Positive & 26 & $17(65.4 \%)$ & $9(34.6 \%)$ & \\
\hline LVI & & & & 0.800 \\
\hline None (0) & 180 & $73(40.6 \%)$ & $107(59.4 \%)$ & \\
\hline
\end{tabular}




\begin{tabular}{|c|c|c|c|c|}
\hline Focal (1-4) & 53 & $19(35.8 \%)$ & $34(64.2 \%)$ & \\
\hline Moderate (5-9) & 18 & $6(33.3 \%)$ & $12(66.7 \%)$ & \\
\hline Extensive $(\geq 10)$ & 13 & $4(30.8 \%)$ & $9(69.2 \%)$ & \\
\hline LNM & & & & 0.395 \\
\hline Negative & 204 & $76(37.3 \%)$ & $128(62.7 \%)$ & \\
\hline Positive & 60 & $26(43.3 \%)$ & $34(56.7 \%)$ & \\
\hline Parametrium invasion & & & & 0.040 \\
\hline Negative & 242 & $89(36.8 \%)$ & $153(63.2 \%)$ & \\
\hline Positive & 22 & $13(59.1 \%)$ & $9(40.9 \%)$ & \\
\hline Surgical margin & & & & 0.012 \\
\hline Negative & 244 & $89(36.5 \%)$ & $155(63.5 \%)$ & \\
\hline Positive & 20 & $13(65.0 \%)$ & $7(35.0 \%)$ & \\
\hline HPV subtype & & & & 0.108 \\
\hline HPV 16 & 67 & $19(28.4 \%)$ & $48(71.6 \%)$ & \\
\hline HPV 18 & 72 & $25(34.7 \%)$ & $47(65.3 \%)$ & \\
\hline Other subtypes & 18 & $8(44.4 \%)$ & $10(55.6 \%)$ & \\
\hline Not available & 1 & $0(0.0 \%)$ & $1(100.0 \%)$ & \\
\hline Negative & 106 & $50(47.2 \%)$ & $56(52.8 \%)$ & \\
\hline MMR & & & & 0.278 \\
\hline dMMR & 20 & $10(50.0 \%)$ & $10(50.0 \%)$ & \\
\hline pMMR & 244 & $92(37.7 \%)$ & $152(62.3 \%)$ & \\
\hline P16 & & & & 0.000 \\
\hline Negative & 24 & $20(83.3 \%)$ & $4(16.7 \%)$ & \\
\hline Positive & 240 & $82(34.2 \%)$ & $158(65.8 \%)$ & \\
\hline Ki67 & & & & 0.004 \\
\hline$<12.5$ & 65 & $35(53.8 \%)$ & $30(46.2 \%)$ & \\
\hline$\geq 12.5$ & 199 & 67(33.7\%) & $132(66.3 \%)$ & \\
\hline
\end{tabular}

${ }^{a}$ Chi-square test; HPVA, HPV-associated adenocarcinoma; NHPVA, nonHPV-associated adenocarcinoma; LVI: lymph vascular invasion; LNM, lymph node metastasis; MMR: mismatch repair; dMMR: deficient 
mismatch repair; pMMR: proficient mismatch repair; other subtypes: HPV45, HPV16/18, HPV18/45, HPV73/35/81, HPV53/56/66, HPV26/51/82, HPV18/39/59/68.

Table 2. Univariate and multivariate analyses of clinicopathological parameters and TACC3 expression for overall survival( $n=264)$. 


\begin{tabular}{|c|c|c|c|c|}
\hline \multirow[t]{2}{*}{ Variables } & \multicolumn{2}{|l|}{$\begin{array}{l}\text { Univariate } \\
\text { analysis }\end{array}$} & \multicolumn{2}{|c|}{ Multivariate analysis } \\
\hline & $\mathrm{HR}(95 \% \mathrm{Cl})$ & $P$ & $\mathrm{HR}(95 \% \mathrm{Cl})$ & $P$ \\
\hline Age (<37 vs. $\geq 37$ years) & $\begin{array}{l}0.381(0.180- \\
0.802)\end{array}$ & 0.011 & $\begin{array}{l}0.678(0.280- \\
1.639)\end{array}$ & 0.388 \\
\hline Figo stage (I vs. II vs. III vs. IV) & $\begin{array}{l}2.383(1.585- \\
3.581)\end{array}$ & 0.000 & $\begin{array}{l}1.116(0.699- \\
1.782)\end{array}$ & 0.645 \\
\hline Tumor size(cm) (<4.5vs. $\geq 4.5)$ & $\begin{array}{l}2.414(1.222- \\
4.768)\end{array}$ & 0.011 & $\begin{array}{l}1.079(0.487- \\
2.390)\end{array}$ & 0.851 \\
\hline Histological type (HPVA vs. NHPVA) & $\begin{array}{l}0.293(0.138- \\
0.621)\end{array}$ & 0.001 & $\begin{array}{l}0.610(0.228- \\
1.632)\end{array}$ & 0.325 \\
\hline Differentiation (Good vs. Moderate vs. Low) & $\begin{array}{l}1.427(0.803- \\
2.535)\end{array}$ & 0.226 & & \\
\hline Stromal invasion (<1/3 vs. 1/3-2/3vs. $\geq 2 / 3)$ & $\begin{array}{l}3.581(2.004- \\
6.400)\end{array}$ & 0.000 & $\begin{array}{l}1.832(0.953- \\
3.522)\end{array}$ & 0.070 \\
\hline Nerve invasion (Negative vs. Positive) & $\begin{array}{l}3.990(1.884- \\
8.450)\end{array}$ & 0.000 & $\begin{array}{l}0.653(0.246- \\
1.730)\end{array}$ & 0.391 \\
\hline $\begin{array}{l}\text { LVI (None vs. Focal vs. Moderate vs. } \\
\text { Extensive) }\end{array}$ & $\begin{array}{l}2.061(1.550- \\
2.741)\end{array}$ & 0.000 & $\begin{array}{l}1.740(1.200- \\
2.524)\end{array}$ & 0.003 \\
\hline LNM (Negative vs. Positive) & $\begin{array}{l}7.270(3.832- \\
13.794)\end{array}$ & 0.000 & $\begin{array}{l}3.100(1.381- \\
6.959)\end{array}$ & 0.006 \\
\hline Parametrium invasion (Negative vs. Positive) & $\begin{array}{l}5.650(2.581- \\
12.367)\end{array}$ & 0.000 & $\begin{array}{l}1.345(0.514- \\
3.516)\end{array}$ & 0.546 \\
\hline Surgical margin (Negative vs. Positive) & $\begin{array}{l}3.401(1.499- \\
7.716)\end{array}$ & 0.003 & $\begin{array}{l}1.123(0.430- \\
2.934)\end{array}$ & 0.813 \\
\hline $\begin{array}{l}\text { HPV subtype (HPV } 16 \text { vs. HPV18 vs. Other } \\
\text { types vs. Not available vs. Negative) }\end{array}$ & $\begin{array}{l}0.845(0.607- \\
1.177)\end{array}$ & 0.321 & & \\
\hline MMR (dMMR vs. pMMR) & $\begin{array}{l}0.918(0.283- \\
2.981)\end{array}$ & 0.886 & & \\
\hline P16 (Negative vs. Positive) & $\begin{array}{l}0.332(0.152- \\
0.723)\end{array}$ & 0.006 & $\begin{array}{l}0.584(0.214- \\
1.598)\end{array}$ & 0.295 \\
\hline Ki67 (<12.5vs. $\geq 12.5)$ & $\begin{array}{l}0.614(0.316- \\
1.196)\end{array}$ & 0.152 & & \\
\hline TACC3 (Low vs. High) & $\begin{array}{l}2.727(1.440- \\
5.165)\end{array}$ & 0.002 & $\begin{array}{l}2.280(1.087- \\
4.783)\end{array}$ & 0.029 \\
\hline
\end{tabular}

HR, hazard ratio; $\mathrm{Cl}$, confidence interval. HPVA, HPV-associated adenocarcinoma; NHPVA, nonHPVassociated adenocarcinoma; LVI: lymph vascular invasion; LNM, lymph node metastasis; MMR: 
mismatch repair; dMMR: deficient mismatch repair; pMMR: proficient mismatch repair; other subtypes: HPV45, HPV16/18, HPV18/45, HPV73/35/81, HPV53/56/66, HPV26/51/82, HPV18/39/59/68.

Table 3. Univariate and multivariate analyses of clinicopathological parameters and TACC3 expression for Disease-free survival $(n=264)$. 


\begin{tabular}{|c|c|c|c|c|}
\hline \multirow[t]{2}{*}{ Variables } & \multicolumn{2}{|l|}{$\begin{array}{l}\text { Univariate } \\
\text { analysis }\end{array}$} & \multicolumn{2}{|c|}{ Multivariate analysis } \\
\hline & $\mathrm{HR}(95 \% \mathrm{Cl})$ & $P$ & $\mathrm{HR}(95 \% \mathrm{Cl})$ & $P$ \\
\hline Age (<37 vs. $\geq 37$ years) & $\begin{array}{l}0.530(0.266- \\
1.056)\end{array}$ & 0.071 & & \\
\hline Figo stage (I vs. II vs. III vs. IV) & $\begin{array}{l}1.941(1.330- \\
2.832)\end{array}$ & 0.001 & $\begin{array}{l}1.055(0.690- \\
1.614)\end{array}$ & 0.804 \\
\hline Tumor size(cm) (<4.5 vs. $\geq 4.5)$ & $\begin{array}{l}2.303(1.280- \\
4.145)\end{array}$ & 0.005 & $\begin{array}{l}1.138(0.581- \\
2.228)\end{array}$ & 0.707 \\
\hline Histological type (HPVA vs. NHPVA) & $\begin{array}{l}0.310(0.162- \\
0.592)\end{array}$ & 0.000 & $\begin{array}{l}0.536(0.241- \\
1.192)\end{array}$ & 0.126 \\
\hline Differentiation (Good vs. Moderate vs. Low) & $\begin{array}{l}1.266(0.778- \\
2.062)\end{array}$ & 0.343 & & \\
\hline Stromal invasion (<1/3 vs.1/3-2/3 vs. $\geq 2 / 3)$ & $\begin{array}{l}2.363(1.557- \\
3.585)\end{array}$ & 0.000 & $\begin{array}{l}1.468(0.908- \\
2.373)\end{array}$ & 0.117 \\
\hline Nerve invasion (Negative vs. Positive) & $\begin{array}{l}2.846(1.426- \\
5.680)\end{array}$ & 0.003 & $\begin{array}{l}0.659(0.275- \\
1.581)\end{array}$ & 0.350 \\
\hline LVI (None vs. Focal vs. Moderate vs. Extensive) & $\begin{array}{l}1.600(1.214- \\
2.108)\end{array}$ & 0.001 & $\begin{array}{l}1.367(0.975- \\
1.917)\end{array}$ & 0.070 \\
\hline LNM (Negative vs. Positive) & $\begin{array}{l}4.631(2.685- \\
7.987)\end{array}$ & 0.000 & $\begin{array}{l}2.665(1.350- \\
5.262)\end{array}$ & 0.005 \\
\hline Parametrium invasion (Negative vs. Positive) & $\begin{array}{l}4.099(1.987- \\
8.456)\end{array}$ & 0.000 & $\begin{array}{l}1.495(0.635- \\
3.523)\end{array}$ & 0.357 \\
\hline Surgical margin (Negative vs. Positive) & $\begin{array}{l}2.687(1.264- \\
5.712)\end{array}$ & 0.010 & $\begin{array}{l}1.019(0.435- \\
2.390)\end{array}$ & 0.965 \\
\hline $\begin{array}{l}\text { HPV subtype (HPV } 16 \text { vs. HPV18 vs. Other } \\
\text { types vs. Not available vs. Negative) }\end{array}$ & $\begin{array}{l}0.846(0.637- \\
1.123)\end{array}$ & 0.247 & & \\
\hline MMR (dMMR vs. pMMR) & $\begin{array}{l}0.969(0.350- \\
2.686)\end{array}$ & 0.952 & & \\
\hline P16 (Negative vs. Positive) & $\begin{array}{l}0.335(0.172- \\
0.652)\end{array}$ & 0.001 & $\begin{array}{l}0.669(0.293- \\
1.530)\end{array}$ & 0.341 \\
\hline Ki67 (<12.5vs. $\geq 12.5)$ & $\begin{array}{l}0.784(0.431- \\
1.426)\end{array}$ & 0.426 & & \\
\hline TACC3 (Low vs. High) & $\begin{array}{l}2.621(1.517- \\
4.530)\end{array}$ & 0.001 & $\begin{array}{l}2.265(1.232- \\
4.166)\end{array}$ & 0.009 \\
\hline
\end{tabular}

HR, hazard ratio; $\mathrm{Cl}$, confidence interval. HPVA, HPV-associated adenocarcinoma; NHPVA, nonHPVassociated adenocarcinoma; LVI: lymph vascular invasion; LNM, lymph node metastasis; MMR: 
mismatch repair; dMMR: deficient mismatch repair; pMMR: proficient mismatch repair; other subtypes: HPV45, HPV16/18, HPV18/45, HPV73/35/81, HPV53/56/66, HPV26/51/82, HPV18/39/59/68.

\section{Figures}

\section{A}
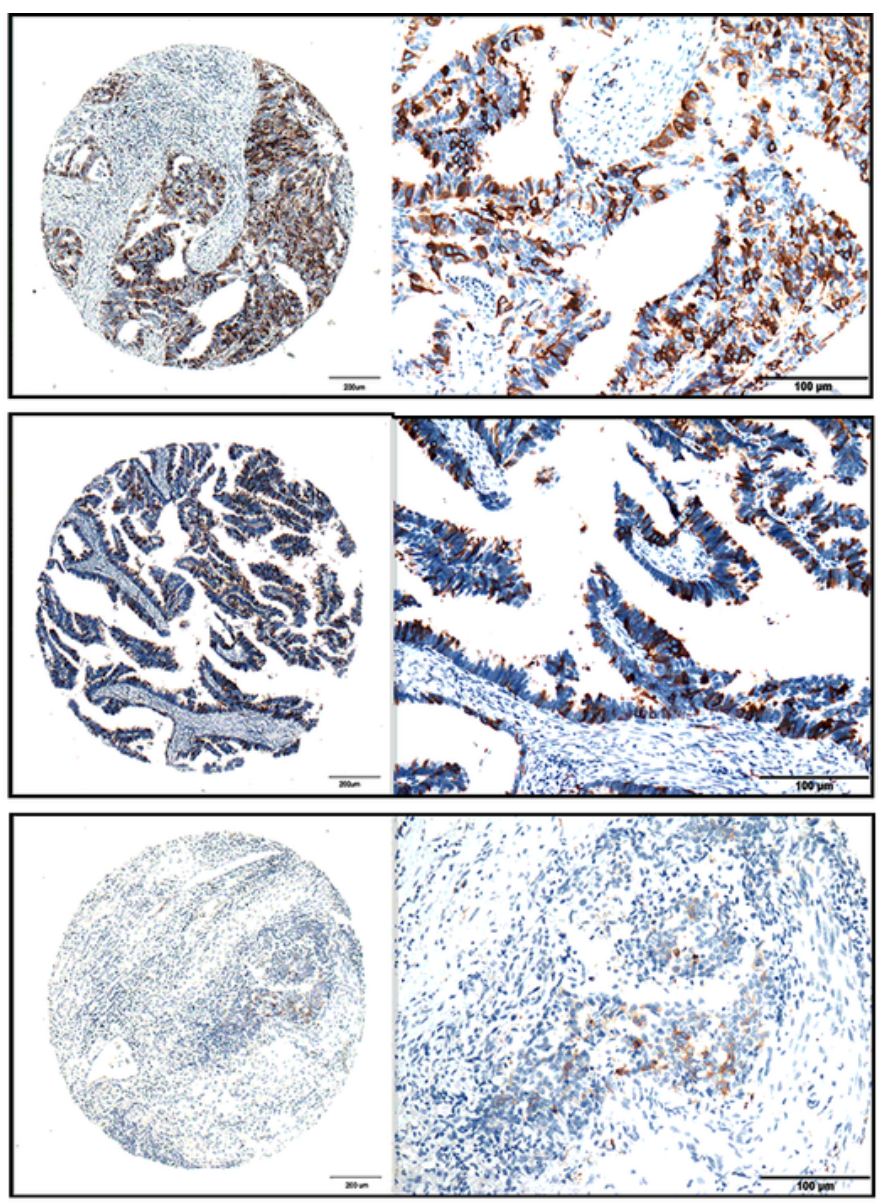

D
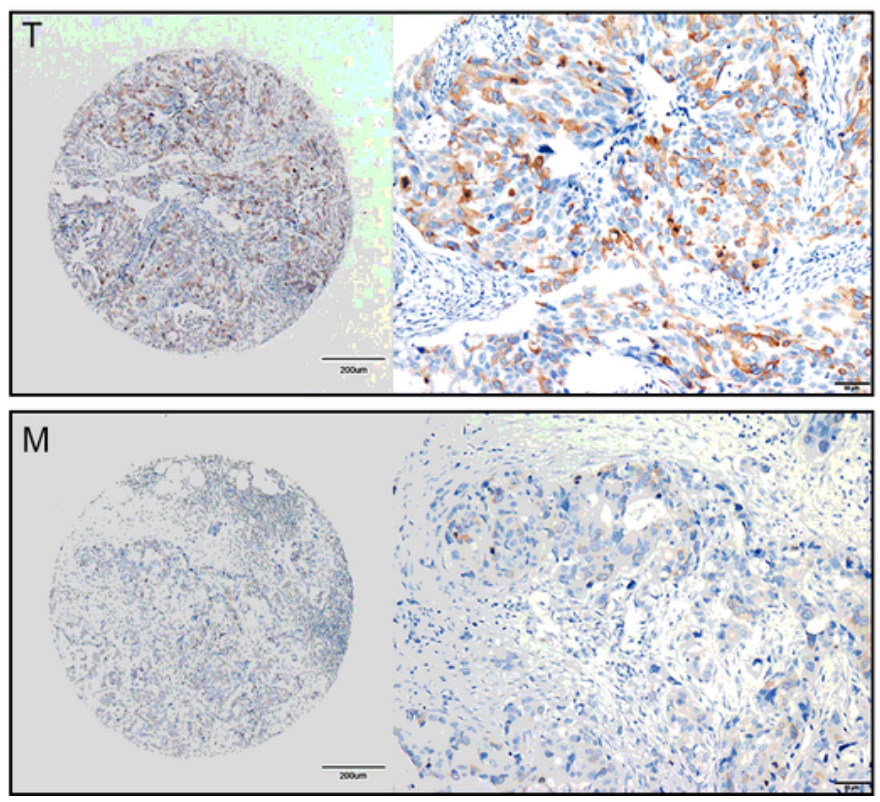
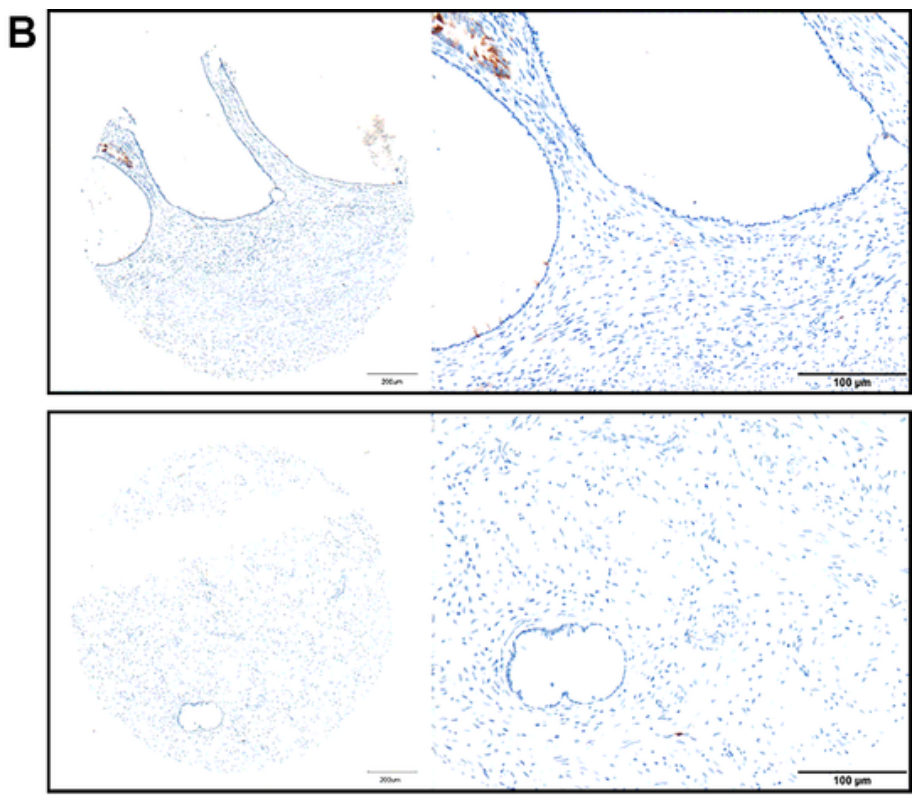

C

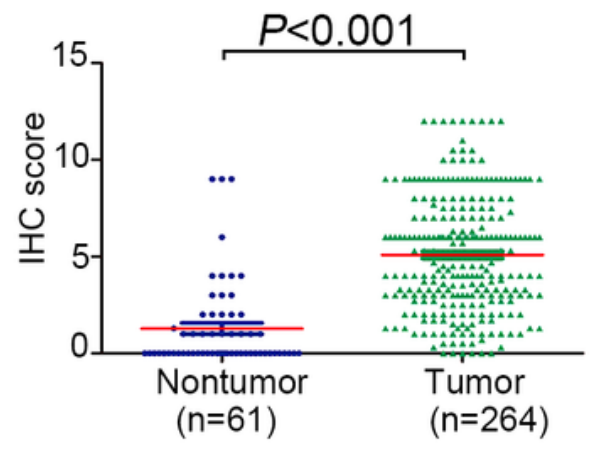

$E$

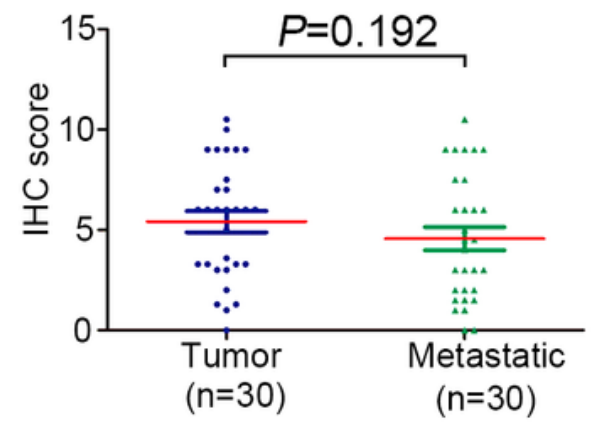

Figure 1 
Overexpression of TACC3 in ECA detected by IHC. A. Representative images of IHC staining for TACC3 expression in a TMA cohort. Representative images of strong, moderate and weak intensity staining for tumor tissues are shown. B. Representative IHC images of positive and negative non-tumor tissues are presented. C. The IHC scores of the TMA cohort, TACC3 expression in ECA was remarkably higher than that in non-tumorous tissues. D. TACC3 expression in 30 ECA with LNM cases analyze by IHC.

Representative graphs are shown for the primary tumor $(T)$ and metastatic $(M)$ lesions. E. Comparison of TACC3 levels between the primary tumor and metastatic lesions. Quantitative data are presented as mean $\pm S D$.
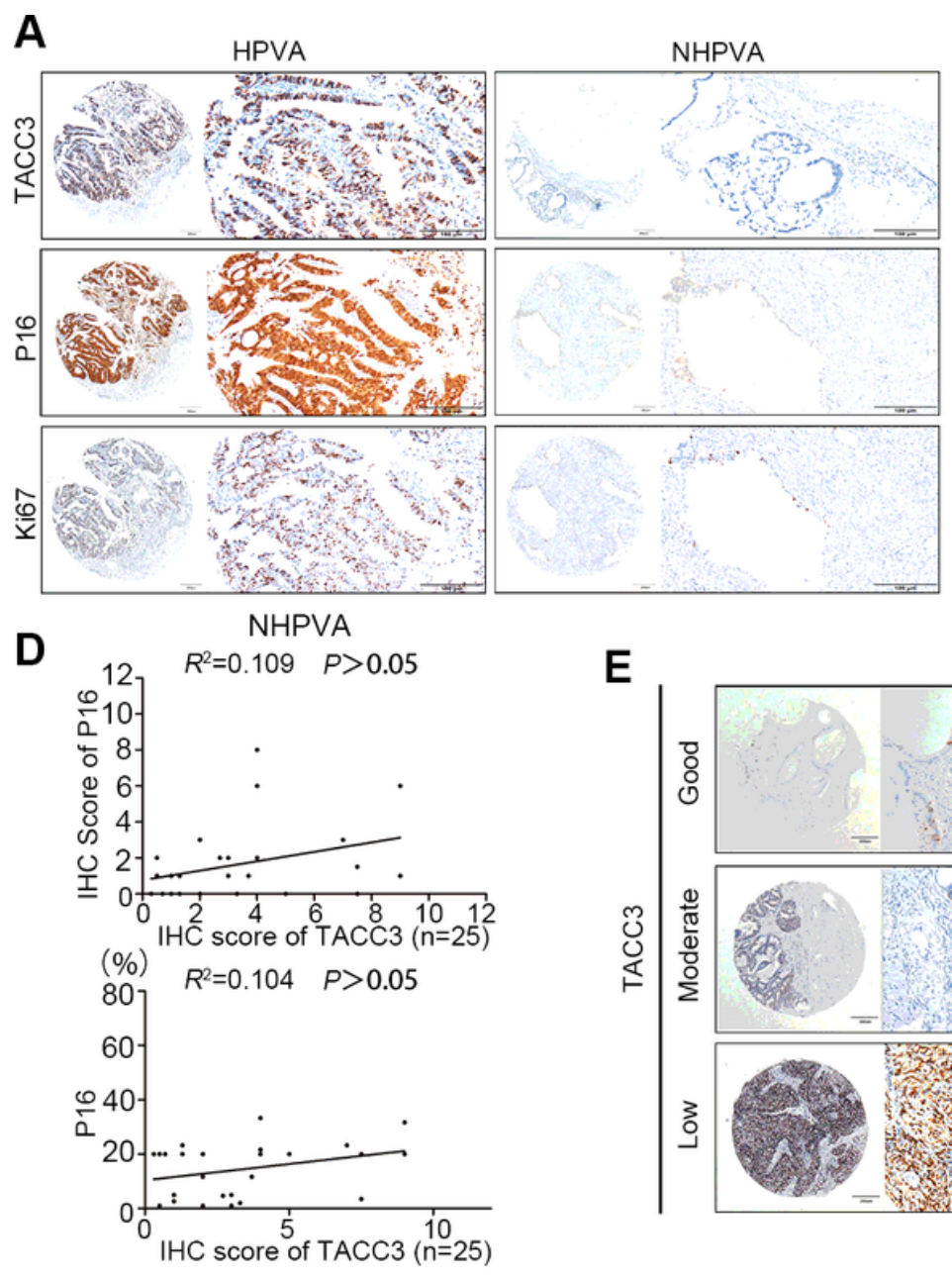
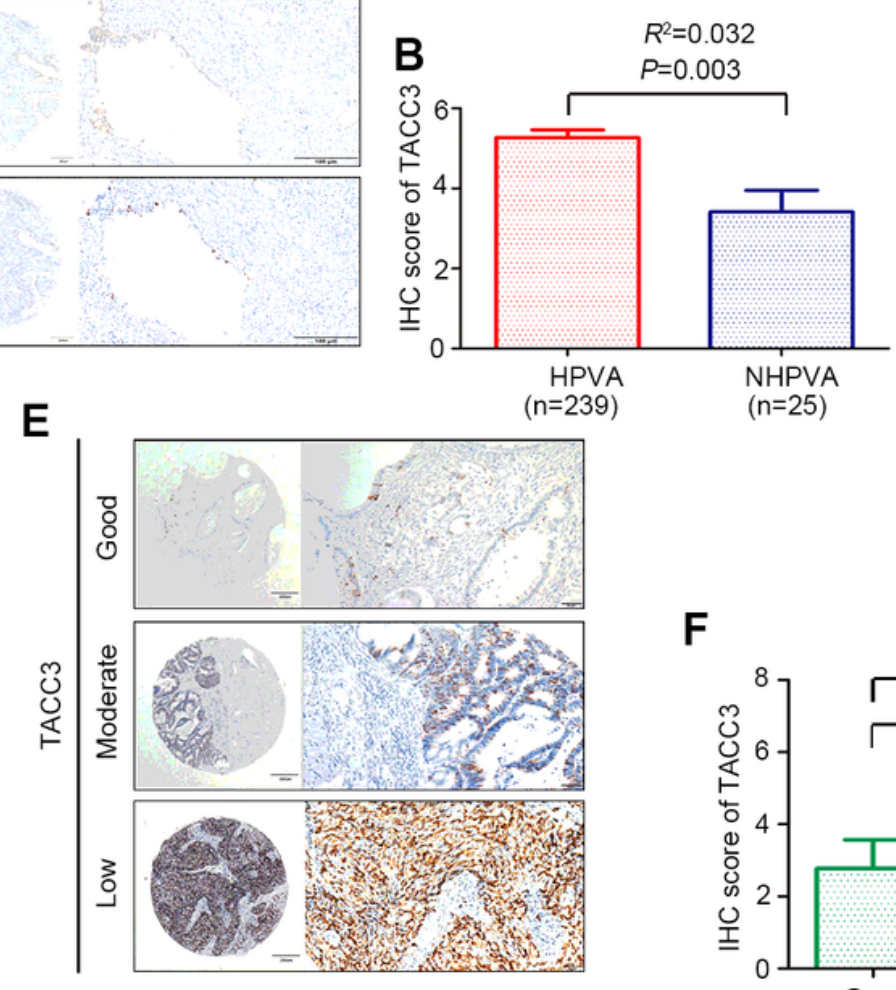
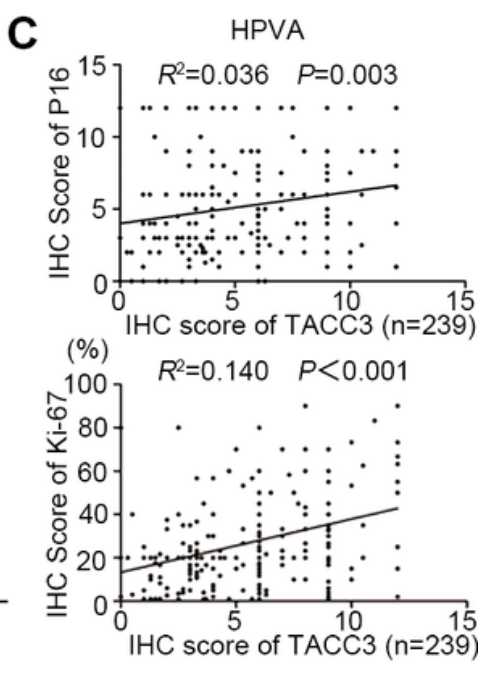

\section{Figure 2}

Relationship of TACC3, P16 and Ki-67 in HPVA and NHPVA. A. Representative images of IHC staining for TACC3, P16 and Ki-67 expression in HPVA and NHPVA. B. The IHC scores of HPVA and NHPVA, TACC3 expression in HPVA was remarkably higher than that in NHPVA. C. The relationship between TACC3 and P16, TACC3 and Ki-67 in HPVA are presented. D. The relationship between TACC3 and P16, TACC3 and Ki67 in NHPVA are presented E. Representative images of TACC3 expression in good-, moderate- and lowdifferentiation tumors are shown. E. Comparison of TACC3 levels in good-, moderate- and lowdifferentiation tumors. Quantitative data are presented as mean \pm SD. 
A

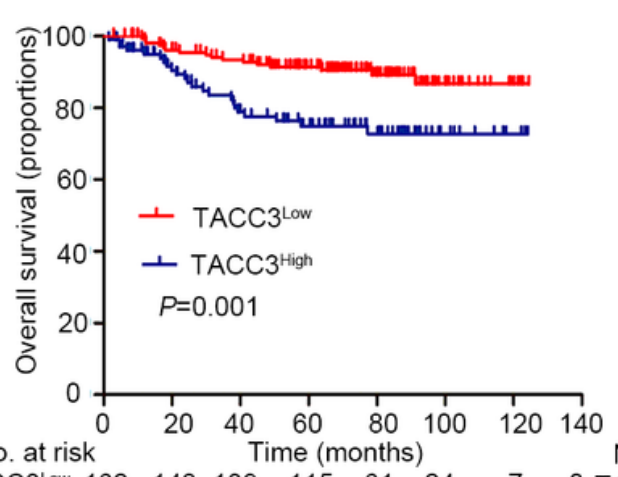

B

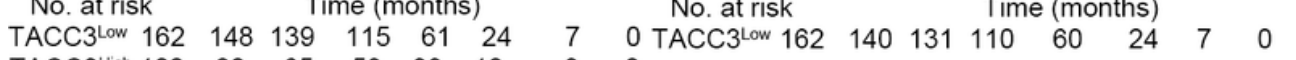

$\begin{array}{lllllllllllllll}\text { TACC3 } 3^{\text {High }} 102 & 80 & 65 & 53 & 33 & 10 & 3 & 0_{\text {TACC3 }}^{\text {High }} 102 & 70 & 60 & 51 & 32 & 9 & 3 & 0\end{array}$

D

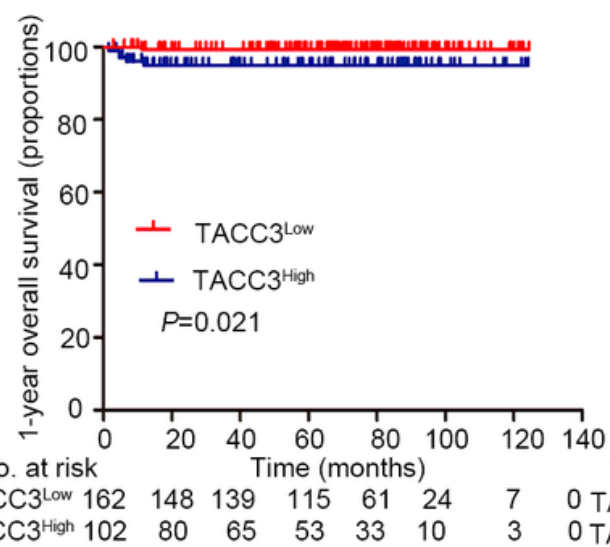

E

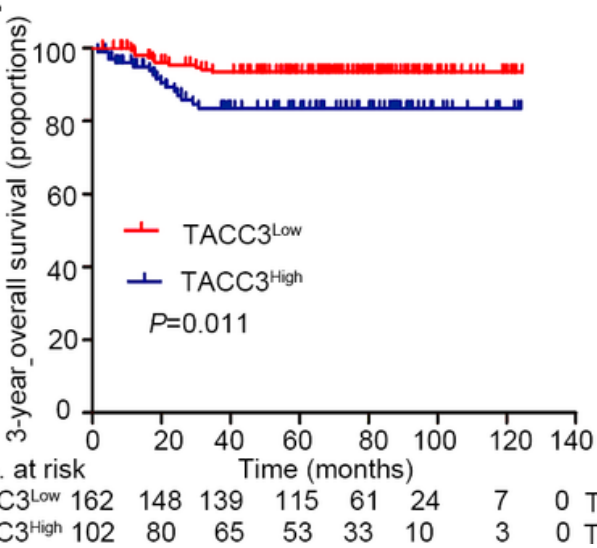

C

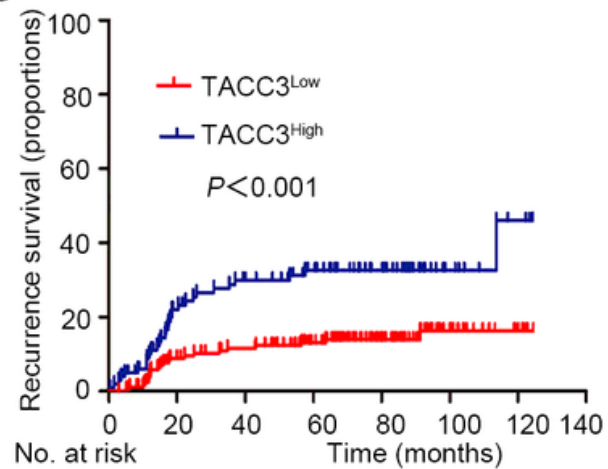

TACC3Low $162140131 \quad 110 \quad 60 \quad 24 \quad 7 \quad 0$

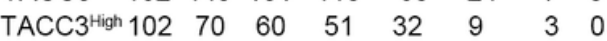

$\mathbf{F}$

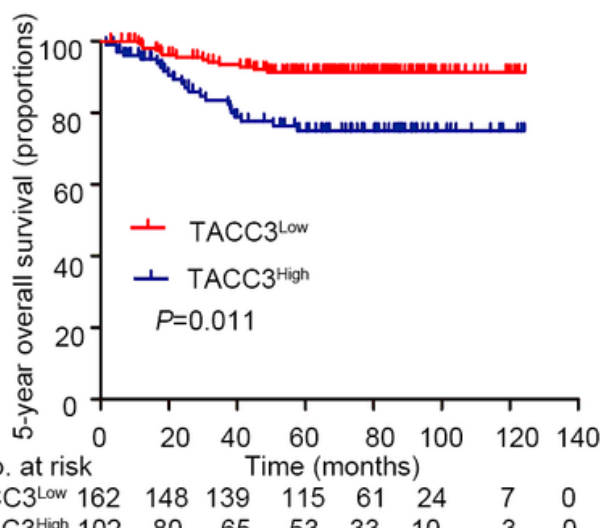

\section{Figure 3}

Association of TACC3 expression and ECA patient survival. A. The correlation of TACC3 expression and overall survival was determined in a TMA cohort including 264 patients by Kaplan-Meier analysis. B. Disease-free survival of the same TACC3 TMA cohort. C. The curve for relapse evaluated according to TACC3 expression level. D. 1-, 3- and 5-year overall survival of the same TACC3 TMA cohort. The life table is shown in each graph below. 
A

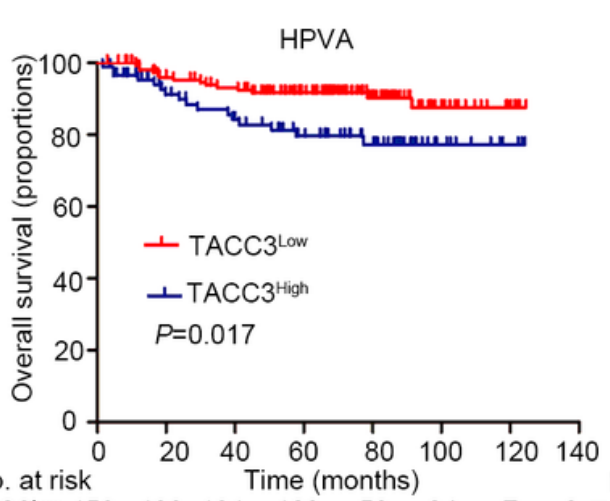

B

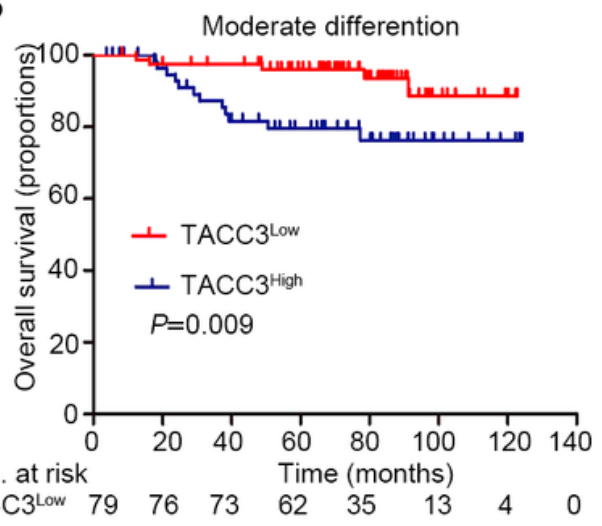
No. at risk $\quad$ Time (months)
C

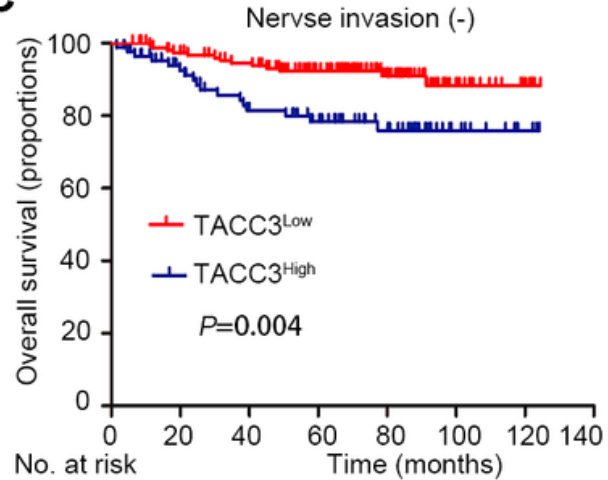
TACC $3^{\text {Low }} 153$

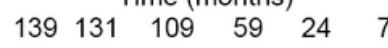

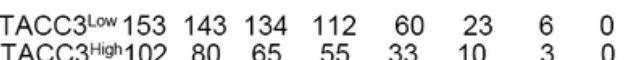

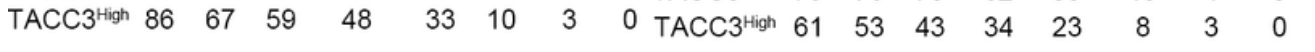

D

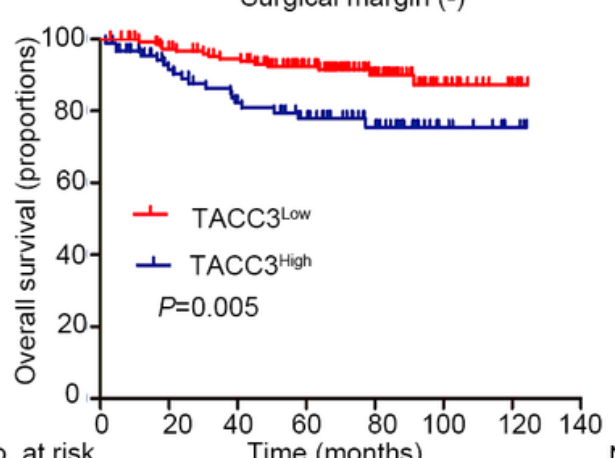

E

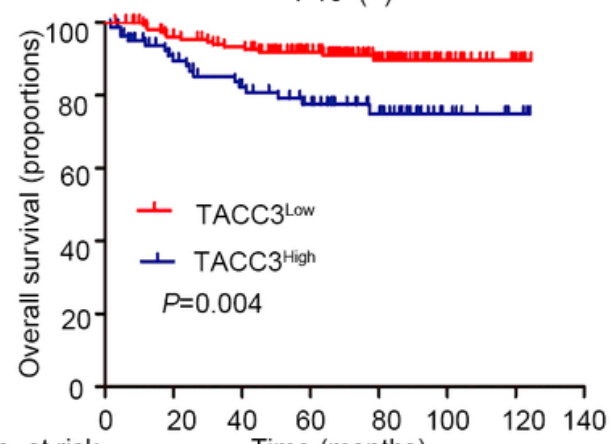

$\mathbf{F}$

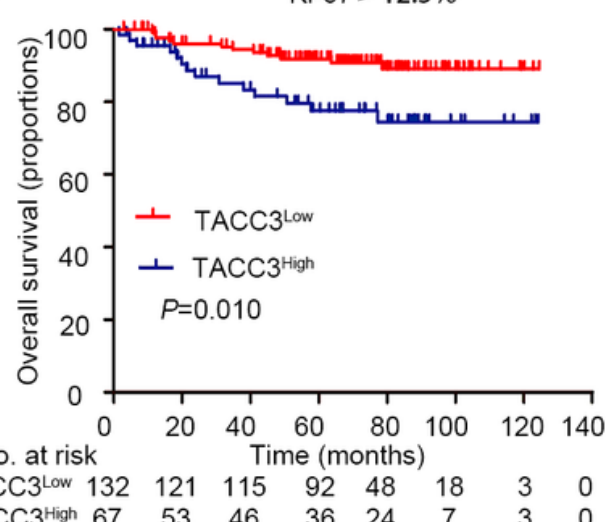

Figure 4

Stratified analysis of TACC3 expression related to overall survival. The correlation of TACC3 expression and overall survival in the indicated groups. 

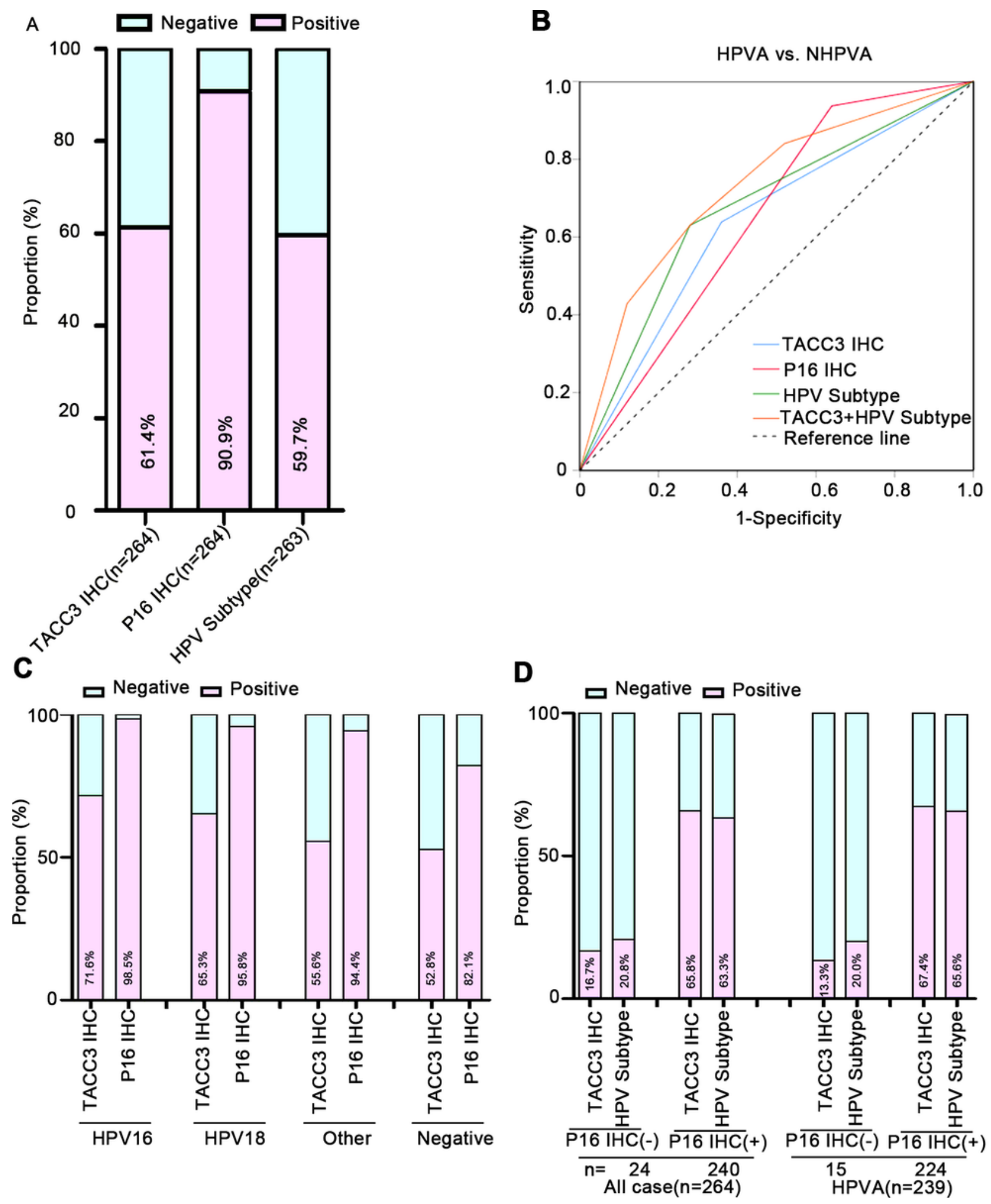

Figure 5

Diagnostic performances of studied testing for ECA patients A. The positive rates of TACC3, P16 and HPV subtypes in ECA cases. B. ROC curve analyses of TACC3, P16, HPV subtypes, and the panel of TACC3+HPV subtypes. C. The positive rates of TACC3 and P16 in ECA cases with different HPV subtype. D. The positive rate of TACC3 and HPV subtype in ECA patients in P16-negative and P16-positive 
subgroups. TACC3 IHC, TACC3 protein in tissues were detected by IHC; P16 IHC, P16 protein in tissues were detected by IHC; HPV subtypes, HPV subtypes were detected by PCR.

\section{Supplementary Files}

This is a list of supplementary files associated with this preprint. Click to download.

- supplementaryfigure1.tif

- SupplementaryFigure2.tif

- SupplementaryTables.docx 